\title{
Machiavélisme et raison d'État dans la tragédie historique : Shakespeare et Chapman
}

\section{Gilles Bertheau}

\section{(QpenEdition \\ 1 Journals}

\section{Édition électronique}

URL : http://journals.openedition.org/shakespeare/759

DOI : 10.4000/shakespeare.759

ISSN : 2271-6424

Éditeur

Société Française Shakespeare

\section{Édition imprimée}

Date de publication : 1 novembre 2002

Pagination : 25-44

\section{Référence électronique}

Gilles Bertheau, « Machiavélisme et raison d'État dans la tragédie historique : Shakespeare et Chapman », Actes des congrès de la Société française Shakespeare [En ligne], 20 | 2002, mis en ligne le 01 novembre 2007, consulté le 01 mai 2019. URL : http://journals.openedition.org/shakespeare/759 ; DOI : 10.4000/shakespeare.759 


\section{S H A K E S P E A R E \& S E S \\ C O N T E M P O R A I N S}

Société Française Shakespeare

Actes du Congrès de 2002

$* * *$

Textes réunis et présentés par

Patricia DORVAL

publiés sous la direction de

Jean-Marie MAGUIN 


\section{MACHIAVÉLISME ET RA IS ON D'ÉTAT DANS LA TRAGÉDIE HISTORIQUE : SHA KES PEARE ET C HAPMAN}

Environ dix années s'écoulent entre l'écriture de Henry IV par William Shakespeare, probablement vers $1597-98$, et celle de The Conspiracy and Tragedy of Byron par George Chapman, vers 1607-08'. Environ deux siècles séparent le règne du Henry IV anglais, qui meurt en 1413, du règne du Henri IV français, qui fait exécuter le maréchal de Biron en 1602. Pourtant les deux dramaturges expriment la même interrogation politique, tous les deux font part d'un même questionnement sur ce qu'est le pouvoir politique.

Mais chacun a son point de vue. L'un se place en Angleterre au début du XVe siècle, dans un royaume qui tente de sortir du système féodal pour devenir un état moderne. L'autre se situe plus loin, en France, mais beaucoup plus près dans le temps, puisque Biron est exécuté en 1602 et qu'Henri IV est encore vivant quand la pièce paraît. La France à cette époque est gouvernée par un souverain moderne qui s'efforce de rompre avec les grands seigneurs tels que Byron.

Mais dans les deux cas, le roi est dépeint sous les traits d'un fin politique qui, faute de pouvoir ramener les rebelles à la raison par son autorité, va employer tous les moyens pour les mettre hors d'état de nuire à sa personne, à son royaume et à l'État en général. Parmi ces moyens, le 
machiavélisme est privilégié et mis paradoxalement au service de la raison d'État, indépendante de toute morale.

Que ce soit face à des rebelles dont les revendications peuvent paraître légitimes mais qui en fait s'opposent à l'avènement d'un système politique moderne, ou bien face à des révoltés calculateurs et impies, l'enjeu est le même, c'est la liberté du monarque, au sens où Jacques $\mathrm{I}^{\text {er }}$ emploie l'adjectif «free» dans The Trew Law of Free Monarchies, traité contemporain de Henry IV. Il entend par là être libre de toute tutelle, qu'elle soit religieuse ou seigneuriale, comme c'est le cas dans les pièces envisagées. C'est pourquoi il est logique que la figure du monarque moderne émerge finalement avec l'affirmation du droit divin et du caractère solaire du roi, comme Shakespeare et Chapman le montrent.

Dans chaque pièce, les poètes ont marqué une différence entre deux types de rebelles : les aristocrates soldats et les aristocrates politiques. Hotspur et ses acolytes (son père le comte de Northumberland, son oncle Thomas Percy, Edmund Mortimer, le comte de Douglas, Owen Glendower et Sir Richard Vernon) et le duc de Byron font partie de cette première catégorie. L'ingratitude royale semble être leur premier sujet de mécontentement, mais c'est davantage contre un État qui menace de se passer d'eux qu'ils s'élèvent.

Le thème de l'ingratitude royale est central dans l'une et l'autre pièce. Quelle que soit la réalité de cette ingratitude, les rebelles ressentent une injustice à leur endroit et s'estiment mal récompensés de l'aide apportée au monarque. Ainsi, après que le roi a fait un portrait sinistre de Mortimer, Hotspur déclare : «I will lift the down-trod Mortimer / As high in the air as this unthankful King, / As this ingrate and canker'd Bolingbroke» (1 Henry IV, I.3.133-5). Byron, de son côté, rappelle les combats menés pour sauver son roi quand il dit : «The fights, the dangers, the affrights and horrors, / Whence I have rescued this unthankful King» (The Conspiracy, V.1.132-3), en employant exactement la même expression.

Aucun des rebelles ne manque de rappeler au roi ce qu'il leur doit. Or la notion de dette, qui implique l'acceptation par les deux parties d'un lien d'obligation mutuel, ne peut plus avoir cours dans un royaume où le monarque cherche précisément à s'émanciper des liens d'obligation qui l'attachent à ses nobles. Ainsi Hotspur dit-il à Blunt, le messager royal : 
«My father, and my uncle, and myself / Did give him that same royalty he wears» (1 Henry IV, IV.3.54-5). Puis il énonce ses griefs :

Hot. In short time after he depos'd the King,

Soon after that depriv'd him of his life, [...]

Disgrac'd me in my happy victories ${ }^{2}$,

Sought to entrap me by intelligence,

Rated mine uncle from the Council-board,

In rage dismiss'd my father from the court, $[. .$.

And in conclusion drove us to seek out

This head of safety, and withal to pry

Into his title, the which we find

Too indirect for long continuance.

$$
\text { (1 Henry IV, IV.3.90-1 ; 97-100; 102-5) }
$$

Worcester tient le même discours au roi, et lui rappelle quelle aide lui et sa famille lui ont apportée afin qu'il obtienne le trône : «It was myself, my brother, and his son, / That brought you home, and boldly did outdare / The dangers of the time» (1 Henry IV, V.1.38-40).

Le mécontentement de ces nobles est très proche de l'attitude d'un Byron outré par l'ingratitude du roi, qui vient de lui refuser le commandement d'une citadelle frontalière :

\author{
Byr. I will see \\ That all your chronicles be filled with me, \\ That none but I and my renownèd sire \\ Be said to win the memorable fields \\ Of Arques and Dieppe, [...] \\ Nor none but I at Fountaine François burst \\ The heart-strings of the leaguers ; I alone \\ Took Amiens in these arms and held her fast [...].
}

(The Conspiracy, V.1.137-41; 145-7)

Mise à part la démesure propre à Byron, les propos sont très similaires à ceux de Worcester et de Hotspur, qui considèrent que le roi ne les a pas récompensés comme ils le méritent. En ce sens, on peut dire que ce sont des Malcontents, le terme «discontented» est utilisé à plusieurs reprises par Chapman ${ }^{3}$.

Or, si chez Shakespeare, les motifs de rébellion peuvent sembler assez légitimes, du moins pour Hotspur et ses compagnons, chez Chapman 
ils sont beaucoup plus douteux car Byron a été largement récompensé par le roi, qui ne manque pas de le rappeler au début de The Tragedy:

Hen. Byron fallen in so trait'rous a relapse

Alleged for our ingratitude! What offices,

Titles of honour, and what admiration

Could France afford him that it poured not on ?

(The Tragedy, I.1.1-4)

Puis Henry rappelle la glorieuse carrière du baron de Byron, devenu duc, pair et maréchal de France en quelques années, par le seul fait de sa faveur.

Au-delà de l'ingratitude royale, le vrai motif de la rébellion de part et d'autre est le malaise que ressentent les grands seigneurs face à un monarque qui tend à s'émanciper de leur pouvoir. Arlette Jouanna et Lawrence Stone en ont chacun bien rendu compte dans respectivement $L e$ Devoir de révolte et The Crisis of Aristocracy ${ }^{4}$. Les grands seigneurs se rendent compte qu'ils deviennent inutiles, surtout en temps de paix. Byron s'en plaint amèrement quand il déclare fort lucidement :

\footnotetext{
Byr. Since Bretagne is reduced, and breathless war

Hath sheathed his sword and wrapped his ensigns up,

The king hath now no more use of my valour, And therefore I shall now no more enjoy

The credit that my service held with him [...].
}

(The Tragedy, I.2.5-9)

Ils sont au contraire supplantés par les courtisans et les ministres. Dans Byron, le roi est entouré de son chancelier, Pomponne de Bellièvre, de Janin, ministre, ainsi que de capitaines et de gardes qui tous à leur niveau représentent l'État. Le système se met en place dans Henry IV, comme en témoignent ces paroles du roi envers Worcester, qu'il congédie sans ménagement : "You have good leave to leave us ; when we need / Your use and counsel we shall send for you» (1 Henry IV, I.3.19-20). Les grands, si nombreux autour du Henry IV anglais, ne sont plus nécessaires au gouvernement de son royaume, ils deviennent, au contraire, facultatifs, voire tout à fait encombrants. 
C'est donc à un courtisan que le roi confie la tâche de réclamer les prisonniers de Hotspur. Or, Hotspur comme Byron déteste cette nouvelle race de nobles. Il suffit d'entendre le récit de l'arrivée de ce seigneur par Henry Percy pour comprendre le fossé qui sépare ces deux hommes :

Hot. When I was dry with rage, and extreme toil, Breathless and faint, leaning upon my sword, Came there a certain lord, neat and trimly dress' $d,[\ldots]$ He was perfumed like a milliner $[\ldots]$.

$$
\text { (1 Henry IV, I.3.30-2; 35) }
$$

Le reste du discours met en avant les traits féminins du courtisan, exact opposé de la valeur chevaleresque et virile déployée par Hotspur et par Byron, symbolisée par leur épée et leurs blessures. Ainsi Hotspur défend-il Mortimer devant le roi en insistant sur ses blessures :

Hot. He never did fall off, my sovereign liege,

But by the chance of war : to prove that true Needs no more but one tongue for all those wounds, Those mouthed wounds, which valiantly he took [...].

(1 Henry IV, I.3.93-6)

Le terme de «liege» employé par Hotspur est significatif du lien entre souverain et vassal. Byron, au cours de son procès, n'hésite pas non plus à rappeler le sacrifice de son sang, versé pour le roi :

Byr. This body, gash'd with five and thirty wounds,

Whose life and death you have in your award, Holds not a vein that hath not open'd been, And which I would not open yet again For you and yours [...].

(The Tragedy, V.2.207-11)

L'image de la saignée est d'ailleurs utilisée par Hotspur : «Yea, on his part [Mortimer's] I'll empty all these veins, / And shed my dear blood, drop by drop in the dust» (1 Henry IV, I.3.131-2).

Mais au fond, le combat mené par les rebelles est dirigé contre un État moderne ou en passe de le devenir. Cela est assez clair chez Chapman, qui fait dire à Byron : 


$$
\begin{aligned}
& \text { Byr. Like th'ancient gods } \\
& \text { Are modern kings, that lived past bound themselves, } \\
& \text { Yet set a measure down to wretched men ; } \\
& \text { By many sophisms they made good deceit, } \\
& \text { And since they passed in power, surpassed in right. }
\end{aligned}
$$

(The Tragedy, V.3.59-63)

Outre l'accusation de machiavélisme qu'un mot comme «deceit» (vers 62) permet de déceler, Byron accuse implicitement le roi de tyrannie quand il dit : «past bound themselves» (vers 60) et «surpassed in right» (vers 63). L'adjectif «modern» lui-même, qui n'apparaît pas dans Henry $I V$, est chargé de connotations machiavéliques. Innocent Gentillet l'emploie comme un terme extrêmement péjoratif : «Mais maintenant par le gouvernement italien et moderne, les bonnes et anciennes loix du royaume sont abolies et aneanties, les guerres cruelles sont entretenues en France, les paix rompues, le peuple ruiné et mangé, le commerce aneanty»".

Hotspur et Byron méprisent donc ce qui ressemble à du machiavélisme, qu'ils savent par ailleurs identifier comme tel. Hotspur en défendant l'honneur de Mortimer le lave de tout soupçon de machiavélisme, en employant le terme si caractéristique de "policy» ${ }^{6}$ :

Hot. Never did bare and rotten policy

Colour her working with such deadly wounds,

Nor never could the noble Mortimer

Receive so many, and all willingly :

Then let not him be slander'd with revolt.

(1 Henry IV, I.3.93-6; 107-11)

Byron aussi rejette le machiavélisme :

Byr. $\quad[\ldots]$ there are schools

Now broken ope in all parts of the world,

First founded in ingenious Italy,

Where some conclusions of estate are held

That for a day preserve a prince, and ever

Destroy him after ; from thence men are taught

To glide into degrees of height by craft

And then lock in themselves by villany. 
Malgré ce que veut faire croire Edward Grimeston, la source de Chapman pour Byron, le maréchal est tout sauf machiavélien'. S'il est certes coupable de trahison - Chapman ne le nie pas - il est également innocent par naïveté politique ${ }^{-8}$. Il est coupable de comploter contre le roi avec des puissances étrangères catholiques (l'Espagne et la Savoie), mais innocent d'un point de vue politique car, aveuglé par une ambition démesurée, il se fait entièrement manipuler par le duc de Savoie, le vrai machiavel de la pièce.

Le but des deux rébellions semble être de fonder un ordre nouveau après une période de chaos, à laquelle aspire Northumberland : «Now let not Nature's hand / Keep the wild flood confin'd! Let order die !» (2 Henry IV, I.1.153-4). Lord Bardolph, de son côté, précise au début de 2 Henry IV : «this great work - / Which is almost to pluck a kingdom down / And set another up» (2 Henry IV, I.3.48-50). Byron de son côté ne déclare-t-il pas vouloir créer un nouveau monde : «on our chaos / Will I sit brooding up another world» (The Tragedy, 1.2.30-1)?

Cette volonté démiurgique ne fait qu'ajouter au crime que constitue déjà la résistance au souverain. La rébellion contre un monarque sacré par l'onction ne peut être que condamnée, car elle n'est pas légale. C'est ce que Blunt rappelle à Hotspur, Worcester, Douglas et Vernon dans une formule lapidaire: «You stand against anointed majesty» (1 Henry IV, IV.3.40). La rébellion est également synonyme de désordre intérieur, ce que le roi ne peut tolérer: "You conjure from the breast of civil peace / Such bold hostility» (1 Henry IV, IV.3.43-4).

Henry quant à lui parle des actions de Byron en employant les expressions «lawless resolutions» (The Tragedy, I.1.52), «unnatural conspiracy» (The Tragedy, I.3.30), ce qui prouve que toute révolte va contre le droit et l'ordre naturel.

De fait, ces personnages s'élèvent contre une longue tradition, celle de l'obéisssance et de l'ordre telle qu'elle est prêchée dans les sermons élisabéthains, comme ceux de 1559 et de 1570. Le premier, An Exhortation Concerning Good Order, and Obedience to Rulers and Magistrates, avertit : «it is not lawful for Inferiors and Subjects, in any case to resist and stand against the Superior Powers : for Saint Pauls words be plain, that whosoever withstandeth, withstandeth the ordinance of God» ${ }^{9}$. Dans le second, An Homily Against Disobedience and Wylful Rebellion, qui complète et renforce le premier, on peut lire : «such subjects as are disobedient or rebellious against their princes disobey God, and procure 
their own damnation» ${ }^{10}$. Le devoir des sujets se décline donc de deux manières : obéir au roi et ne pas désobéir au roi".

Jacques $\mathrm{I}^{\mathrm{er}}$ de son côté ne dit rien d'autre quand il commente le premier livre de Samuel dans The Trew Law of Free Monarchies (1598) : «will ye consider the very wordes of the text in order, [...] it shall plainly declare the obedience that the people owe to their King in all respects» ${ }^{12}$.

L'autre visage de la révolte est politique et met en scène des personnages d'une autre nature. Chez Chapman, c'est le duc de Savoie et La Fin qui remplissent ce rôle, chez Shakespeare, c'est l'archevêque d'York. Ces trois personnages révèlent un des visages du machiavélisme, celui qui a été véhiculé par Gentillet, dont la traduction par Simon Patericke paraît la même année que la pièce de Chapman, en 1608.

Savoy, La Fin et York sont les vrais machiavels des deux pièces. Ils sont tous adeptes de la manipulation des esprits : Savoy et La Fin envers Byron, York envers le peuple, bien qu'il ne soit qu'un sujet du roi anglais, alors que le duc de Savoie est un prince souverain. D'ailleurs, dans The Conspiracy - le seul volet où il apparaît - Savoy tient le deuxième rôle après Byron, mais devant Henry.

Soucieux de récupérer son marquisat de Saluces, conquis par Henry, il vient à la Cour de France sous le prétexte de rencontrer le monarque. Mais en fait, Savoy, allié à l'Espagne, tente de déstabiliser Henry IV et de rallier Byron à son complot en lui promettant la souveraineté sur la Bourgogne. Naturellement, c'est dans le secret que se mènent toutes ces tractations, au point même que ses conseillers ignorent le véritable but de son voyage :

Sav. My hope in France you know not, though my counsel ; [...] Oft-times princes' rules Are like the chymical philosophers'; Leave me then to mine own projection In this our thrifty alchemy of state [...].

(The Conspiracy, I.1.50; 52-5)

La métaphore alchimique employée par le duc pour désigner l'activité politique renvoie à l'idée d'une science politique moderne qui, outre la pratique du pouvoir qu'elle implique, suppose des connaissances 
particulières réservées à des initiés. Elle met en outre l'accent sur la manipulation des passions à laquelle se livre Savoy envers Byron : il va exploiter son orgueil extrême pour créer un conflit entre lui et le roi, à propos des mérites militaires du maréchal, point extrêmement sensible s'il en est. Le duc de Savoie va les exagérer auprès du roi (The Conspiracy, II.2.66-240), de sorte que ce dernier en prendra ombrage, réaction que Savoy s'empressera de rapporter à Byron : «All this shall to the duke : I fished for this» (The Conspiracy, II.2.241). En ce sens, Savoy suit de près la maxime que Gentillet extrait de Machiavel : «Un prince doit savoir cavaller les esprits des hommes pour les tromper» (III, $19^{\mathrm{e}}$ maxime).

La Fin est encore plus nettement caractérisé comme le vilain machiavélique, par ses actions et par la description qu'en fait Henry IV quand il le chasse de sa Cour par exemple :

Hen. Thou witchest with thy smiles, suckst blood with praises, Mockst all humanity, society poisonst, [...].

A man must think of all the villanies

He knows in all men to decipher thee, That art the centre to impiety.

(The Conspiracy, I.1.154-5 ; 159-61)

Tout à la fois hypocrite, fourbe, méchant et impie, ce personnage diabolique incarne donc le machiavel par excellence, tel que l'a dépeint Gentillet, qui insiste sur l'impiété du Florentin : «Machiavel [...] s'est montré par ses escrits un vray atheiste et contempteur de toute pieté» ${ }^{\text {'3 }}$. Les termes de «suckst» et «poisonst» renvoient aussi à Gentillet, qui parle des Italiens, «qui par leur avarice active [...] tondent la laine sur le dos, et nous succent le sang et la substance, comme on feroit à des moutons» ${ }^{14}$ et compare Machiavel aux empoisonneurs «qui ne jettent jamais gros lopin de poison sur un morceau, afin qu'elle ne soit apperceuë, mais l'incorporent le plus subtilement qu'ils peuvent avec quelques morceaux frians et delicats» '. Diabolique, il l'est si on en croit Henry, qui déclare : «La Fiend, and not La Fin, he should be called» (The Conspiracy, III.2.226).

York, le meneur de la révolte dans 2 Henry $I V$, apporte sa caution religieuse à une cause politique. Ce faisant, il se conduit comme un prince qui met la religion au service de la politique, comme Machiavel le montre à propos des Romains, en donnant l'exemple de Numa Pompilius, que 
reprend Gentillet: «Numa Pompilius [...] s'avisa qu'il faloit necessairement inventer quelque belle religion, bien ornee et paree de belles ceremonies, parce que sans religion il luy sembloit impossible de maintenir police entre les hommes» (III, $9^{e}$ maxime) ${ }^{16}$. La présentation que Morton, un des rebelles pourtant, fait de l'archevêque, est sans ambiguité :

\author{
Mor. But now the Bishop \\ Turns insurrection to religion ; \\ Suppos'd sincere and holy in his thoughts, \\ He's follow'd both with body and with mind, $[\ldots]$ \\ Derives from heaven his quarrel and his cause [...].
}

(2 Henry IV, I.1.200-3 ; 206)

Il a percé à jour l'intention de l'archevêque et le caractère faussement providentiel («suppos'd sincere and holy», vers 202) de sa démarche".

L'autre reproche des rebelles au roi est de ne pas avoir accès à sa personne : «When we are wrong'd, and would unfold our griefs, / We are denied access unto his person» (2 Henry IV, IV.1.77-8), où on retrouve le désir des nobles d'être proches du centre du pouvoir, au moment même où le roi veut les en éloigner.

Mais Westmoreland rejette ces arguments :

Wes. $\quad[\ldots]$ it not appears to me

Either from the King or in the present time That you should have an inch of any ground To build a grief on $[\ldots]$.

(2 Henry IV, IV.1.107-10)

Et il met au jour la mauvaise foi de l'archevêque :

Wes. Whenever yet was your appeal denied ? [...] That you should seal this lawless bloody book Of forg'd rebellion with a seal divine, And consecrate commotion's bitter edge?

(2 Henry IV, IV.1.88 ; 91-3)

Le prince Jean y voit en outre une tentative de manipulation des sujets du roi, que l'archevêque a voulu soulever («up-swarm'd», vers 30) contre leur monarque : 
Lan. $\quad \mathrm{O}$, who shall believe

But you misuse the reverence of your place, Employ the countenance and grace of heav'n, As a false favourite doth his prince's name, In deeds dishonourable? You have ta'en up, Under the counterfeited zeal of God, The subjects of his substitute, my father, And both against the peace of heaven and him Have here up-swarm'd them.

(2 Henry IV, IV.2.22-30)

Les termes «suppos'd», vu plus haut, ainsi que «countenance» (vers 24), «false» (vers 25) et «counterfeited» (vers 27) marquent bien le machiavélisme d'York en ce qu'ils insistent sur la tromperie comme arme politique.

L'argument religieux masque mal les véritables motifs de la révolte. York accuse la nécessité d'être la cause de leur conduite, il accuse la corruption des temps présents, viciés par la paix et le bonheur :
Arc. We are all diseas'd,
And with our surfeiting, and wanton hours,
Have brought ourselves into a burning fever,
And we must bleed for it ; [...]
And purge th' obstructions which begin to stop
Our very yeins of life.

(2 Henry IV, IV.1.54-7; 65-6)

Cette litanie familière qui accuse la paix de tous les maux se trouve aussi chez Byron :

Byr. O the most base fruits of a settled peace !

In men, I mean ; [...] [they]

Grow rude and foggy, overgrown with weeds,

Their spirits and freedoms smothered in their ease,

And as their tyrants and their ministers

Grow wild in prosecution of their lusts [...].

(The Tragedy, IV.1.1-2; 9-12) 
Ce thème est le thème favori des machiavels de théâtre, comme Baligny dans The Revenge of Bussy D'Ambois de Chapman, par exemple.

Mais York, en fait, profite de l'occasion qui se présente, comme il le dit : "We see which way the stream of time doth run, / And are enforc'd from our most quiet there / By the rough torrent of occasion» (2 Henry IV, IV.1.70-2). Un peu de la même façon que Bolingbroke s'est emparé du pouvoir : «then, God knows, I had no such intent [to ascend the throne] / But that necessity so bow'd the state / That I and greatness were compell'd to kiss» (2 Henry IV, III.1.72-4). Shakespeare, par cet effet de miroir, dresse un parallèle frappant entre les méthodes des deux personnages.

Ces révoltes vont donner l'occasion aux monarques considérés de faire valoir la raison d'État, servie par un machiavélisme que rejetait pourtant Giovanni Botero, l'auteur de Della Ragion di Stato en 1589.

Force, parjure, ruse : tels sont les instruments dont les deux monarques se servent pour venir à bout de ceux qui leur résistent. Ces trois moyens forment ce qu'on peut appeler le machiavélisme apparent des deux monarques.

Chez Shakespeare, Henry utilise les armes contre les rebelles dans un conflit interne, qui implique aussi l'Écosse cependant. À la fin de la première partie, Vernon et Mortimer sont mis à mort sans autre forme de procès, le roi épargnant les comtes de Northumberland et de Worcester. Byron aussi est mis à mort ${ }^{13}$, mais après un procès qui a toutes les apparences de la légalité.

Le parjure est l'élément essentiel de la victoire du roi sur les rebelles dans la seconde partie de la pièce de Shakespeare. Après avoir accepté la liste des doléances des seigneurs en armes contre lui, le roi, par l'intermédiaire de son cadet, le prince Jean, promet solennellement de redresser les torts et de faire retirer son armée :

Lan. My Lord, these griefs shall be with speed redress'd ;

Upon my soul they shall. If this may please you, Discharge your powers unto their several counties, As we will ours $[\ldots]$.

(2 Henry IV, IV.2.60-2) 
York lui répond: «I take your princely word for these redresses» (2 Henry IV, IV.2.66). Une fois l'armée adverse débandée et la sienne toujours en place, le prince peut faire arrêter les trois conspirateurs, qui l'accusent immédiatement de parjure : «Will you thus break your faith ?» demande l'archevêque (2 Henry IV, IV.2.112), «I pawn'd thee none», répond le prince (2 Henry IV, IV.2.112), qui rompt ainsi avec le code d'honneur des aristocrates au nom du salut de l'État ${ }^{\prime \prime}$.

Le parjure n'est qu'un des aspects de la ruse, qui, dans Byron, s'exprime d'une manière différente. Après avoir chassé La Fin de sa Cour, dans The Conspiracy, Henry le rappelle auprès de lui et s'en sert d'agent double pour piéger Byron, dans The Tragedy:

Hen. Good de La Fin, you were our golden plummet

To sound this gulf of ingratitude ;

In which you have with excellent desert

Of loyalty and policy expressed

Your name in action [...].

(The Tragedy, I.3.10-4)

C'est grâce à La Fin et à ses propos rassurants que le maréchal rentre à la Cour, après s'être réfugié en Bourgogne. En cela, La Fin s'est montré fidèle aux ordres du roi, qui lui avait dit :

Hen. And good de La Fin, I pray you write to him

To hasten his repair, and make him sure

That you have satisfied me to the full

For all his actions, and have uttered nought

But what might serve to banish bad impressions.

(The Tragedy, II.3.91-5)

Or c'est à la Cour que Byron est arrêté et c'est à cause du témoignage de La Fin lors du procès qu'il est condamné à mort. Le tout orchestré par un roi en apparence impeccable, mais qui n'hésite pas à recourir aux services d'un homme qu'il avait violemment fustigé quelques scènes auparavant.

De fait, ni le Henry français, ni le Henry anglais ne sont exempts de défauts. Mais à la différence du premier, qui se protège derrière des procédures légales, le second ne fait pas mystère de son machiavélisme. 
Roi légal arrivé au pouvoir par usurpation et régicide envers Richard II, Henry Bolingbroke dit à son fils, le prince Hal :

Kin. God knows, my son, By what by-paths and indirect crook'd ways I met this crown, and I myself know well How troublesome it sat upon my head.

(2 Henry IV, IV.5.183-6)

Tandis que le Bourbon, roi légal et légitime, se pose en monarque respectueux de la justice, comme il le dit à ses ministres après l'arrestation du maréchal :

Hen. I like not executions so informal, For which my predecessors have been blamed [...]. The decent ceremonies of my laws And their solemnities shall be observed, With all their sternness and severity.

(The Tragedy, IV.2.39-40; 45-7)

On notera déjà que le terme de «ceremonies» laisse place à l'ambiguïté.

Ce machiavélisme royal est perçu par leurs sujets. Hotspur parle ainsi de Bolingbroke en disant «this subtle King» (1 Henry IV, 1.3.167), «this vile politician Bolingbroke» (l Henry IV, I.3.238) et en l'appelant «this king of smiles» (1 Henry IV, I.3.243). Le terme «subtle» marque le mépris du jeune Percy envers le roi. Gentillet aussi dans son livre emploie le nom «subtilité» associé à la «perfidie» : «Revenons maintenant à nostre propos des subtilitez que nous disons ne devoir estre pratiquees au gouvernement des affaires d'estat, et qu' on ne doit par icelles couvrir une perfidie» (III, $21^{\mathrm{e}}$ maxime) ${ }^{20}$.

Le roi français de son côté est appelé «this politic King» par le duc de Savoie (The Conspiracy, I.1.86). Byron déclare quant à lui : «My gloss [...] / Had too much splendour for the owly eye / Of politic and thankless royalty» (The Tragedy, V.2.181-3).

L'attitude des deux souverains face aux aristocrates rebelles est similaire : ils veulent les éloigner du pouvoir. Après avoir découvert sa tentative de trahison, Henry envoie Byron en Angleterre auprès d'Élisabeth $\mathrm{I}^{\text {ère }}$ tandis que l'Anglais avoue à son héritier se méfier des 
nobles et lui conseille de les tenir occupés, de peur qu'ils ne se mêlent de trop près des affaires de l'État :

Kin. And all my friends, which thou must make thy friends,

Have but their stings and teeth newly ta'en out ;

By whose fell working I was first advanc'd, And by whose power I well might logde a fear

To be again displac'd ; which to avoid,

I cut them off, and had a purpose now

To lead out many to the Holy Land,

Lest rest and lying still might make them look

Too near unto my state. Therefore, my Harry,

Be it thy course to busy giddy minds

With foreign quarrels, that action hence borne out

May waste the memory of the former days.

(2 Henry IV, IV.5.204-15)

Cela permet au roi de conserver la haute main sur le royaume et de se passer de barons frondeurs et encombrants. On est ici au cœur du machiavélisme, compris comme l'autonomisation du pouvoir politique du prince.

Ce que les deux dramaturges mettent en scène n'est autre que la raison d'État en marche. Énoncée comme telle chez Chapman par le ministre Janin, qui explique à Henry :

Jan. Princes, you know, are masters of their laws, And may resolve them to what forms they please, So all conclude in justice [...] [...] the great mother

Of all productions, grave Necessity, Commands the variation; and the profit, So certainly foreseen, commends the example.

(The Tragedy, IV.2.31-3; 35-8)

Au-delà de la transgression de la loi morale que représentent le parjure et la tromperie, la raison d'État, interprétée par ce personnage, propose ainsi la transgression de la loi positive. Botero, traduit par Gabriel Chappuys, définit la raison d'État ainsi : «Estat est vne ferme domination sur les peuples ; \& la Raison d'Estat est la cognoissance des moyens propres à 
fonder, conseruer, \& agrandir vne telle domination \& Seigneurie» («Quelle chose est la Raison d'Estat») ${ }^{21}$. Tout tient dans les moyens employés pour maintenir l'État : pour ces deux dramaturges, qui se situent moins du point de vue de ce qui doit être que de ce qui est, il semble bien que le machiavélisme soit le moyen, sinon le plus recommandable, du moins le plus efficace. Mais quand Shakespeare montre le fonctionnement de l'État tel qu'il le perçoit, de manière neutre en apparence, Chapman révèle les abus d'un État absolutiste naissant. Il n'est pas innocent qu'il se place du point de vue de Byron, comme le titre de la pièce en témoigne, plutôt que du point de vue du roi, qui donne son nom à la pièce de Shakespeare. La figure d'Henry chez Chapman est beaucoup plus ambiguë qu'il n'y paraît et Byron beaucoup moins coupable qu'il n'en a l'air, (beaucoup moins coupable en tout cas que York) même si le dramaturge ne le dédouane pas de ses actes ${ }^{22}$.

Enfin, il n'est pas anodin que cette affirmation puissante de l'autorité soit accompagnée dans les deux cas d'une affirmation concomitante du droit divin des rois et de la nature solaire du monarque. Ce droit est nié par Hotspur, pour qui Bolingbroke n'est que le fruit d'une élection, sur le mode féodal. S'adressant à ses compagnons, il dit : «you that set the crown / Upon the head of this forgetful man» (1 Henry IV, I.3.158-9) ${ }^{23}$.

Il est donc primordial pour ces rois, s'ils veulent être véritablement souverains, «libres» au sens jacobéen du terme, de se présenter comme les élus de Dieu seul, comme ses «substituts» pour employer le mot du prince Jean (2 Henry IV, IV.2.28). Le Henry français déclare quant à lui, au début de The Tragedy: «My rule is threatened; and that sacred power / [...] will not see / A trait'rous subject foil me» (The Tragedy, I.1.99; 105-6).

Chez Shakespeare on voit se dessiner la figure d'un prince chrétien dans les paroles du prince Jean. En réponse à York qui l'accuse de parjure, il répond : «I promis'd you redress of these same grievances / Whereof you did complain ; which, by mine honour, / I will perform with a most Christian care» (2 Henry IV, IV.2.113-5). De plus son père reprend l'idée de croisade qu'il avait émise au début de la pièce.

Enfin, le parti royal ne manque pas d'arracher aux rebelles l'approbation providentielle qu'ils avaient usurpée : «God, and not we, hath safely fought today», dit le prince ( 2 Henry IV, IV.2.121), après que son père eut déclaré, au début de 1 Henry IV : «God befriend us as our cause is just !» (1 Henry IV, V.1.120). Dans le même temps, il reconnaît qu'il doit sa couronne à l'opinion, en partie du moins : «Opinion, that did help me to the crown» (1 Henry IV, III.2.42). On peut donc suggérer que 
l'interprétation providentielle de la victoire royale n'est qu'un signe ultime de son machiavélisme.

L'affirmation du droit divin va de pair avec l'utilisation de la métaphore solaire par les monarques. Ainsi Henry, après l'arrestation de Byron déclare-t-il : «Now I am settled in my sun of height, / The circular splendour and full sphere of state / Take all place up from envy [...]» (The Tragedy, V.1.138-40). Le prince Jean dit: «That man that sits within a monarch's heart, / And ripens in the sunshine of his favour» (2 Henry IV, IV.2.11-2). Ces images aussi sont employées par le futur Henry V: «Two stars keep not their motion in one sphere, / Nor can one England brook a double reign / Of Harry Percy and the Prince of Wales» (I Henry IV, V.4.64-66).

Comme le dit le prince Harry, il ne peut y avoir deux étoiles au ciel d'Angleterre. De même, il ne peut y avoir deux sources du pouvoir dans un État moderne. C'est ce que Chapman et Shakespeare ont compris : «les rois modernes» ne peuvent ni ne veulent plus longtemps partager leur autorité avec quiconque, y compris et surtout les plus grands seigneurs, dont la fronde contre l'absolutisme royal ne finit pas avec ces deux pièces, et qui mettent en pratique un machiavélisme assumé pour affirmer leur liberté souveraine.

Gilles Bertheau Université de Versailles Saint-Quentin-en-Yvelines

\section{NOTES}

'Les éditions utilisées sont les suivantes: William Shakespeare, $1 \&$ 2 Henry IV, éd. A.R. Humphreys, Londres, Methuen \& Co, 1960 et 1966 ; George Chapman, The Conspiracy and Tragedy of Charles Duke of Byron, éd. John Margeson, Manchester, Manchester University Press, 1988. Cette dernière pièce 
étant en deux parties, il sera fait référence respectivement à The Conspiracy et à The Tragedy par forme d'abréviation.

${ }^{2} \mathrm{Cf}$. ce que dit Machiavel à ce propos: «Ce capitaine qui, avec tant de valeur, a conquis à son maître un État; qui, par ses victoires sur l'ennemi, s'est couvert de gloire [...] ; ce capitaine acquiert nécessairement parmi ses soldats et ceux de l'ennemi, et parmi les sujets du prince, une si haute renommée qu'elle cesse d'être du goût de son maître. [...] Le prince ne peut donc alors que songer à s'assurer du capitaine ; et pour cela, ou il s'en défait, ou il cherche à le discréditer dans l'armée, parmi le peuple, en s'efforçant de persuader que sa victoire est moins le fruit de sa virtù que du bonheur ou de la lâcheté de ses ennemis, ou de la virtù des autres officiers qui ont combattu avec lui» (Discours sur la première décade de Tite-Live, I, 29) Euvres complètes, éd. Edmond Barincou, Paris, Gallimard Pléiade, 1952, p. 445-6.

${ }^{3}$ Cf. The Conspiracy, I.1.11 et 95 ; The Tragedy, I.2.41.

${ }^{4}$ Arlette Jouanna, Le Devoir de révolte : la noblesse française et la gestation de l'État moderne (1559-1661), [Paris], Fayard, 1989. Lawrence Stone, The Crisis of Aristocracy 1558-1641, Oxford, Clarendon Press, 1965.

'Innocent Gentillet, Anti-Machiavel, éd. C. Edward Rathé, Les Classiques de la pensée politique 5, réimpr. de Discours svr les moyens de bien gouverner... Contre Nicolas Machiavel Florentin (1576), Genève, Librairie Droz, 1968, p. 39.

'Napoleone Orsini, «'Policy' or the Language of Elizabethan Machiavellism», Journal of the Warburg and Courtauld Institute, $n^{\circ} 9,1946$, p. 122-34.

${ }^{7}$ Edward Grimeston, traducteur ici de Pierre Matthieu, écrit : «Hee should have knowne that Machiavels councell (who saith that private men never rise from a base to a high fortune but by fraude and force) is ruinous [...]», A Generall Historie of France, Written by Iohn de Serres vnto the Yeare 1598. Much Augmented and Continued unto this Present, out of the Most Approoued Authors That Have Written of That Subiect, 1607, Londres, George Eld, 1611, p. 1115.

${ }^{8}$ Il ne peut pas imaginer, par exemple, que La Fin, en qui il a placé toute sa confiance, puisse le trahir pour le roi. «Can all the world / Make him a treacher ?» demande-t-il avec stupéfaction quand La Fin s'apprête à témoigner contre lui à son procès (The Tragedy, V.2.124-5).

'David Wootton, éd., Divine Right and Democracy : An Anthology of Political Writing in Stuart England, Londres, Penguin Books, 1986, p. 65.

${ }^{10}$ Op. cit., p. 96-7.

"On remarquera le passage, entre 1559 et 1570 , d'une exhortation à l'obéissance à une exhortation contre la désobéissance. Cela peut s'expliquer par la rébellion du Nord en faveur de Marie Stuart (1569-70) et par la publication en février 1570 de la bulle d'excommunication d'Élisabeth $\mathrm{I}^{\mathrm{èr}}$ (Regnans in excelsis) 
du pape Pie $\mathrm{V}$, qui appelle les sujets anglais à désobéir à une reine considérée par Rome comme une usurpatrice.

${ }^{12}$ Jacques I $I^{\text {er }}$, The Political Works of James I, éd. Charles Edward Macllwain, 1616, Cambridge (USA), Harvard U.P., 1918, p. 58.

${ }^{13}$ Innocent Gentillet, Anti-Machiavel, Préface de la première partie, p. 41.

${ }^{14}$ Op. cit., p. 43.

${ }^{15}$ Op. cit., p. 33-4.

${ }^{16}$ «La religion de Numa fut la principale cause de la félicité de Rome», $O p$. cit., p. 257.

${ }^{12}$ Cf. Gisèle Venet : «Autour de l'Archevêque d'York, c'est une opposition idéologique au régime qui se constitue et prend les trait usurpés d'une 'mission providentielle', celle de l'Église [...]. Ce qui avait été médiocre calcul d'intérêts de caste devient complot subversif, manipulation idéologique d'un peuple méprisé», Temps et vision tragique : Shakespeare et ses contemporains, Paris, Publications de l'Université de la Sorbonne-Nouvelle, 1985, ch. VI, p. 277.

${ }^{18}$ Contrairement à son complice, le comte d'Auvergne, demi-frère d'Henriette d'Entraygues, maîtresse du roi.

${ }^{19}$ On notera que le parjure constitue un des critères fondamentaux qui ont fait d'Henri III un tyran aux yeux de ses adversaires, comme l'Advertissement des Novvelles crvautez \& inhumanitez, desseignees par le Tyran de la France en témoigne : «lon ne peut plus faire aucun doute que la Declaration du Roy de Nauarre ne fust veritable, quand il a dit que par le massacre commis en la ville de Blois, \& rupture de la foy publique, le Tyran auoit pris la querelle pour luy», Paris, Rolin Thierry, 1589, p. 7.

${ }^{20}$ «Le prince prudent ne doit observer la foy, quand l'observation luy en est dommageable, et que les occasions qui la luy ont fait promettre sont passees», op. cit., p. 474.

${ }^{21}$ Giovanni Botero, Raison et Govvernement d'Estat, en dix livres, 1589, trad. Gabriel Chappuys, Paris, Guillaume Chavdiere, 1599, p. 4.

${ }^{22}$ Le Henri IV historique était familier avec le concept de raison d'État, comme en atteste cet extrait de sa correspondance adressée à Monsieur de Beaumont, son ambassadeur en Angleterre (6 mars 1605) : «Vous accompagnerés encore ce compliment d'un rafraischissement et redoublement des asseurances de mon amitié et du contentement que j'ay eu de celle que le dict duc [Lennox] m'a donnée de la sienne, luy disant sur ce subject ce que vous jugerés estre convenable pour luy faire croire que je me moque de tous les artifices dont l'on use de toutes parts pour donner atteinte à nostre amitié. Car, comme d'un costé je sçay que je ne manqueray jamais à celle que je luy ay promise, parce que j'y suis porté par inclination naturelle et par raison d'Estat, je me promets tout aussy de sa prudence et de la sincerité de sa foy, ayant esprouvé l'une et l'autre, qu'il resistera tousjours aussy constamment aux tentations et inventions de ceulx qui entreprendront d'y 
frapper coup [...]», Recueils des lettres missives de Henry IV, éd. M. Berger de Xivrey, Documents inédits sur l'histoire de France, Paris, Imprimerie Impériale, 1850-1853, vol. 6, p. 359.

${ }^{23}$ L'argument de l'État populaire est repris par les monarchomaques, en particulier François Hotman dans sa Francogallia : «I think 'tis most plain, that the Kings of Francogallia were made such, rather by the Suffrages and Favour of the People, than by any Hereditary Right», in Francogallia, s.1., 1573, ch. VI, p. 42. 